\title{
Apreciación del valor patrimonial de las torres de defensa del litoral valenciano
}

\section{Francisco Juan Vidal}

Instituto Universitario de Restauración del Patrimonio (IRP). Universitat Politècnica de València (UPV), Spain, fjuan@ega.upv.es

\begin{abstract}
The paper refers to the network of watchtowers of the Valencian coast, built during the sixteenth century, and proposes the evaluation of their values as heritage with a conceptual methodology based on an operational tool: the evaluation matrix.
\end{abstract}

Keywords: heritage values, evaluation matrix, surveillance and defense towers.

\section{Introducción}

El artículo se centra en la valoración patrimonial de las torres de defensa del litoral valenciano, levantadas durante el S. XVI, fruto de un proyecto que pretendió establecer una red de torres, cuya principal misión era la de advertir y disuadir los frecuentes ataques de piratas a las localidades próximas a la costa. Felipe II decidió encargarle ese ambicioso proyecto al ingeniero Giovanni Battista Antonelli "il Vecchio", a quien siguieron otros ingenieros de la misma familia. Hoy el conjunto de estas torres forma un importante patrimonio arquitectónico.

La valoración que se propone transciende de la componente material y quiere apuntar a la integridad de su significado cultural actual y pretérito, entendiendo como tal los usos, conocimientos y técnicas que las comunidades, los grupos reconocen como parte integrante de su Patrimonio. Se trata de identificar lo que podríamos denominar "unidad cultural" de estas torres que, partiendo de esta componente inmaterial, engloba todos los objetos y elementos que la forman: documentos, historia, instrumentos, indumentaria, técnicas, oficios vinculados, gastronomía, lugares $\mathrm{y}$, por supuesto, sus arquitecturas. En este sentido será una prioridad metodológica la interdisciplinariedad, como forma de propiciar el diálogo entre las diferentes áreas de conocimiento involucradas.

Se propone un procedimiento sistemático para valorar el bien, basado en la "matriz de valoración" como herramienta conceptual, útil para la identificación tanto de los valores de esta unidad cultural, como de los especialistas capaces de evaluarlos y de los grupos sociales a los que concierne su disfrute. El fin de esta valoración patrimonial no es otro que su entera interpretación cultural. Expresar las potencialidades de integración y de desarrollo cultural ofrecidos por estas torres dispersas, mediante una forma de interpretación capaz de establecer sinergias entre ellas, como parte de un mismo "concepto proyectual". Tal operación permitiría, no sólo comprender de modo más completo el valor de cada torre, sino también encontrar en ellas la presencia de un legado cultural común.

\section{La matriz de valoración}

A partir de la relación de factores y dimensiones enunciados en el punto $13^{\circ}$ del "Documento de 
Nara" para evaluar los valores del patrimonio cultural (Lemaire, R \& Stovel, H; 1994), más de una entidad responsable de la gestión cultural ha recurrido a una matriz conceptual para la valoración de los bienes. Se conoce como "Matriz de Nara" y normalmente consiste en la identificación y descripción de valores que definen la condición patrimonial del bien, cumplimentando las celdas de una matriz configurada con los aspectos o factores en las filas (forma y diseño; materiales y substancia; uso y función; tradiciones y técnicas; localización y contexto; espíritu y sentimientos) y las dimensiones del "valor cultural" en las columnas (artística; histórica; científica; social).

Si los denominados "aspectos" del bien, que tienen que ver con su naturaleza y su existencia, poseen validez constante como categorías propias de los objetos, las dimensiones del valor patrimonial son más interpretables y han venido experimentando un deslizamiento conceptual en las últimas décadas (Muñoz, S; 2003) que permite cuestionar la actualidad de la clasificación original, enunciada en la Conferencia de Nara.

El valor historiográfico del bien, entendido como documento de la historia, sigue siendo considerada una dimensión esencial del valor cultural. Parte del convencimiento de que los bienes patrimoniales poseen información valiosa susceptible de lectura. El objetivo fundamental de cualquier intervención será la salvaguardia de la "autenticidad" del documento, testimonio de la historia. Lógicamente será imprescindible conocer con rigor la historia del bien, utilizando métodos propios de las ciencias humanas (historia, arqueología...). Todas las fases y episodios de transformación del objeto patrimonial se valoran por igual y deberán ser conservadas. La intervención que atiende a esta dimensión documental del valor patrimonial se ciñe a la conservación del "documento", y ha pasado de ser una teoría a ser un método basado en conocimientos científicos. A las ciencias humanas se han ido sumando las ciencias de los materiales (física, química, biología).

Sin embargo las otras dimensiones del valor patrimonial, tales como la artística, la histórico- rememorativa y la socio-cultural, se interpretan hoy como aspectos parciales de una misma dimensión global: el valor de significación. Según él lo que caracteriza el valor de los bienes culturales son ciertos rasgos intangibles y subjetivos, establecidos por la sociedad y no inherentes al objeto. Gozan de una consideración especial porque "significan" algo. Un significado convencional, estimado por un grupo de personas. Simbolizan la "identidad" de un determinado grupo social. Nace de un sentimiento vivo que reside en la gente. Es un valor mutable y susceptible de influencias. La intervención que atiende a esta dimensión significativa tiene por objeto conseguir que el bien "comunique" con eficacia esos significados. Es lo que comúnmente se conoce como restauración, entendida como una acción conceptualmente distinta de la conservación.

En el caso particular del valor de los bienes arquitectónicos, al binomio documento / significado hay que sumar una nueva dimensión: el llamado valor "de uso". Está generalmente asumido que no es posible conservar "vivo" el valor patrimonial de una arquitectura si no se dedica a un uso activo. Además de ser producto de la acción humana (como el resto de bienes culturales), la arquitectura es el espacio donde se produce esa acción. Contiene la vida y esta fue, y debe seguir siendo, su razón de ser. La intervención que atiende a esta dimensión vital tiene por objeto adecuar el bien para un uso. $\mathrm{Si}$ el uso se aprecia como valor, siempre será preferible dedicarlo a un uso que contribuya a comprender su razón de ser. Si no fuera posible, cabría un uso afín, o incluso un uso social, siempre que se propicie la interpretación de aquel uso que motivó su existir.

Así, intervenir en el patrimonio arquitectónico siempre tendrá como finalidad atender de forma ponderada las tres dimensiones de su valor patrimonial (documento, significado, uso):

- Conservar el documento construido

- Restaurarlo para restituir la legibilidad de su significado

- Adecuarlo para un uso que permita comprender su "razón de ser" 
Estas tres dimensiones del valor patrimonial serán las columnas de la nueva matriz de valoración (derivada de la matriz de Nara). Inevitablemente estas dimensiones estarán, en mayor o menor medida, interrelacionadas entre sí. Su separación será, sobre todo, un ejercicio conceptual cuya mayor virtud habrá que buscarla en su utilidad operativa.

\subsection{Identificación de los valores patrimoniales}

Se pretende identificar los valores patrimoniales de la red de torres de defensa del litoral valenciano, aplicando la matriz de valoración, aspecto por aspecto. Pese a que se valora el conjunto de torres, como unidad cultural, en muchos casos procederá la valoración adicional de ciertas componentes singulares y/o locales.

\section{- Aspecto 1. Materia y Substancia}

Por "materia y substancia" se entiende el soporte material o elemental que constituye el cuerpo del bien patrimonial. Atenderá a la ejecución, a las técnicas constructivas, a los materiales y a sus aparejos, a los sistemas tectónicos. Precisará desentrañar la estructura interna de sus fábricas y conocer su proceso ejecutivo y la procedencia de sus elementos. En general estarán capacitados para evaluarlo los técnicos en edificación (arquitect@s, ingenier@s) especializados en historia de la construcción, con la asistencia de especialistas conocedores de la historia, capaces de leer los "documentos construidos" (arqueólog@s, especialistas en estratigrafía).

\begin{tabular}{|l|l|l|l|}
\hline \multicolumn{3}{|c|}{ MATRIZ DE VALORACIÓN DE LOS BIENES CULTURALES } \\
\cline { 1 - 2 } Dimensiones & \multirow{2}{*}{ DOCUMENTAL } & \multirow{2}{*}{ SIGNIFICATIVA } & \multirow{2}{*}{ DE USO } \\
\hline Aspectos & & & \\
\hline 1 MATERIA Y SUBSTANCIA & & & \\
\hline 2 FORMA Y DISEÑO & & & \\
\hline 3 USO Y FUNCIÓN & & & \\
\hline 4 LOCALIZACIÓN Y CONTEXTO & & & \\
\hline 5 TRADICIONES Y TÉCNICAS & & & \\
\hline
\end{tabular}

Fig. 1- Esquema de la Matriz de Valoración.

En su dimensión "documental", el valor residirá en la "legibilidad" de los datos que aporten información sobre su construcción (ejecución, técnicas, materiales, aparejos, sistemas...) y sobre la evolución de la misma, entendida como las diferentes fases históricas por las que ha pasado (reformas, ampliaciones, derribos...). El conocimiento constructivo comprenderá una información común al conjunto de torres y otra información específica propia de cada ejemplar. Los grupos sociales receptores serán, en primer lugar, los estudiosos del tema (especialistas, investigadores...) pero podría llegar a serlo cualquier individuo interesado, siempre que se le facilite la interpretación de los datos con los medios adecuados (divulgación). Un grupo particular dentro de este colectivo podría ser el de los visitantes, con el pertinente proyecto de musealización.
En su dimensión "significativa", las fábricas remiten a una arquitectura heredada del pasado. Es un valor de antigüedad. Depende de que su percepción trasmita satisfactoriamente la idea de que las torres se construyeron en otro tiempo, y reside tanto en el aspecto y estado exterior de las fábricas como en las técnicas que permitieron construirlas y que ilustran la sabiduría de oficios pretéritos. Podrá ser receptor de esta dimensión del valor patrimonial cualquier persona, aunque lo interpretarán con mayor intensidad las gentes cuyo pasado esté vinculado al lugar.

En la dimensión "de uso", el valor de la materia dependerá de la "resiliencia" de las fábricas: su capacidad para ser recuperada y utilizada hoy para un uso activo, sin trasgredir la vocación constructiva y la filosofía tectónica con la que fue concebida. Serán destinatarios de este valor, fundamentalmente, los técnicos encargados 
redactar los posibles proyectos de rehabilitación.

\section{- Aspecto 2. Forma y diseño}

Por "forma y diseño" se entiende la manifestación perceptible de su configuración formal y compositiva. Estará sometida al determinismo de la historia, a los condicionantes de la función y a los imperativos del "gusto". Su interpretación requerirá de un mínimo conocimiento de la evolución de las formas de las torres de defensa a lo largo de la historia. Exige dominio de las reglas en uso, de los trazados y composiciones recurrentes, de los esquemas tipológicos utilizados, de las invariantes estéticas acuñadas por la tratadística, la tradición o la cultura, los precedentes y soluciones tipificadas, y también las cuestiones relativas a la permanencia, estabilidad y factores de cambio (Juan-Vidal, F; 2005). Estarán capacitados para evaluarla 1@s arquitect@s especializad@s en arquitectura militar, con la asistencia de especialistas en Historia del Arte.

En su dimensión “documental”, el valor radicará en su relevancia en relación con las tipologías y las morfologías de las torres de defensa de la época. Las invariantes, las singularidades, las innovaciones respecto a las soluciones establecidas o los elementos característicos serán claves para su identificación. También su adscripción a soluciones tipificadas o recurrentes, propias de la configuración arquitectónica (trazados, esquemas, módulos, métricas...). Pasará por clasificar y calificar las diferentes torres de la red en función de su adscripción a las diferentes familias formales, interpretando sus componentes teóricos, históricos y funcionales. La autoría de los Antonelli y su "manera de hacer" será otra de las señas del valor patrimonial en este campo. Los grupos sociales receptores de esta dimensión del valor serán, nuevamente, los estudiosos (especialistas e investigadores), aunque también podrá llegar a serlo cualquier individuo interesado, siempre que se le facilite su interpretación con medios divulgativos.

En cuanto a la dimensión "significativa", el valor habrá que buscarlo tanto en la cualidad estética como en el poder simbólico de la forma.
En el terreno artístico procederá identificar los factores geométricos y topológicos (orden, simetría, proporción, posición, ritmo, armonía...), así como las variables culturales relativas a los cánones estilísticos y a los problemas del gusto (de lo bello). Por su parte, en el ámbito simbólico, convendrá evaluar la capacidad de la forma para comunicar ideas o conceptos asociados a la arquitectura militar (rotundidad, fortaleza, poder, protección...). La conexión entre los diferentes ejemplares, como nudos de una red territorial, permitirá simbolizar significados sociales de orden superior (unidad, colaboración, ubicuidad...). Podrá ser receptor de esta dimensión del valor patrimonial cualquier persona, aunque lo interpretarán con mayor intensidad las gentes del lugar.

Respecto a la dimensión "de uso", el valor de la forma dependerá de la capacidad de la tipología para alojar satisfactoriamente nuevos usos, sin perder los rasgos que la hacen reconocible: la adaptabilidad funcional del tipo. Los casos de conversión de torre en faro, por mencionar un ejemplo recurrente, no parecen satisfacer esta condición. Cabe mencionar aquí los estándares actuales de habitabilidad, accesibilidad, o seguridad, que en ocasiones precisan la implementación de elementos o instalaciones poco compatibles con la esencia tipológica y formal del bien y que, por este motivo, merecerían ser reconsiderados en beneficio del valor patrimonial. Serán receptores de esta dimensión los usuarios, tanto si son habituales como ocasionales.

\section{- Aspecto 3. Uso y función}

El aspecto "uso y función" no tiene por objeto el continente, sino el "contenido" de los bienes arquitectónicos. Tampoco el significante, sino su "significado". Además de ser producto de una acción humana del pasado, la arquitectura patrimonial es el espacio vital de ese pasado: contuvo la vida. Conserva viva la fuerza de la existencia humana que la concibió y que la aprovechó. No sentimos herederos de la vida que albergó, de las acciones que propició y de las ideas que representó, y ello le confiere poder para evocar. Las torres del litoral cumplieron una función defensiva, tanto para avistar las 
amenazas como para disuadirlas. También pretendieron simbolizar el poder del Estado. Además muchas de ellas fueron alojamiento de guarniciones, y su vivencia existencial forma también parte de la historia del lugar. Técnicas, armamentos, pertrechos, indumentaria... pero también usos, costumbres, historias... serán objeto de estudio, junto a la arquitectura. Estarán capacitados para evaluar el relieve del valor patrimonial en este ámbito, los arquitectos especializados en la historia de la arquitectura militar, formando parte de equipos multidisciplinares con asistencia de historiadores, sociólogos, así como de expertos en poliorcética e ingeniería militar.

En la dimensión "documental", el bien patrimonial tendrá valor en tanto que testigo y testimonio de usos, hechos, acontecimientos y avatares que hayan tenido a estas torres como protagonistas, tanto de forma individual como en su conjunto. Permitirá documentar su historia existencial, que se identificará tanto en la estructura de la red como en determinados rasgos de la obra inmueble de cada una de las torres, así como en otros objetos muebles vinculadas con ellas, se conserven o no en el lugar. Los grupos sociales más directamente receptores de este valor serán las gentes vinculadas, en mayor o menor medida, con la historia del lugar, siempre que se le facilite una rigurosa interpretación de los datos. Otro posible grupo receptor, compatible con el anterior, sería el de los visitantes, en el caso de que se lleve a cabo un proyecto de musealización.

En su dimensión "significativa", el valor patrimonial de estas torres en el aspecto funcional estará relacionado con su capacidad re-memorativa: conjunto de referencias del pasado que un grupo de personas reconocen en ellas como tal. Aquí lo importante no serán tanto los hechos o sucesos, sino sus connotaciones. Generalmente su relieve dependerá de su capacidad para representar la "identidad" de las gentes del lugar. Ser símbolo de lo que los pueblos sienten como un legado común. Expresar, en algún sentido, lo que piensan de sí mismos. Se podría definir como un valor de "iconicidad", de naturaleza inmaterial y convencional. Los semiólogos lo llaman función-signo y, en tanto que bien patrimonial, normalmente predomina sobre la función primaria o de utilidad (Eco, U; 2000). No es un lenguaje. Representa valores abstractos, verbalmente imprecisos. Sólo los individuos afectados, que poseen los códigos de interpretación de esa identidad, son capaces de leer este significado. Hay quien dice que los significados con los que los receptores cargan de valor simbólico a estos bienes, pierden intensidad si se explotan turísticamente (Lowenthal, D; 1985).

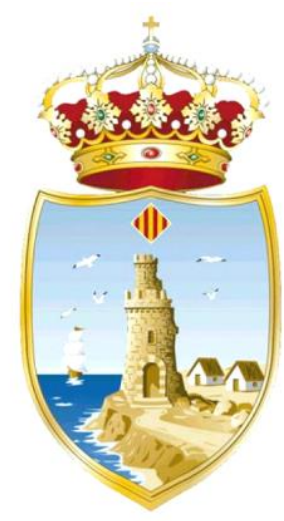

Fig. 2- Escudo de Torrevieja (Alicante).

En cuanto a la dimensión "de uso", el valor patrimonial residirá en la aptitud para permitir la interpretación de sus usos históricos, a partir de los posibles nuevos usos, tanto en su faceta individual como en el papel tuvieron como parte de una red de torres de defensa del litoral. Se podría decir que radicará en su potencialidad para dar a entender cuál fue su verdadera razón de ser, en todas sus dimensiones. Será receptora de esta dimensión del valor patrimonial, la sociedad en general $\mathrm{y}$, con la adecuada divulgación, podría llegar a tener interesantes cualidades didácticas y formativas.

\section{- Aspecto 4. Localización y contexto}

El aspecto "localización y contexto" evalúa la implantación territorial de las torres y su influencia para generar un nuevo "orden" en el lugar. Tratará de descubrir las vinculaciones de estas arquitecturas con las condiciones que impone el medio donde se implantan y con las 
circunstancias de cuando se concibieron. La ubicación es uno de los factores esenciales para comprender las atalayas defensivas, sea a nivel individual o como integrantes de una red, y por lo tanto un aspecto imprescindible para su interpretación como patrimonio. Conllevará, sobre todo, un cambio de escala en los análisis, porque en las relaciones arquitectura / lugar siempre conviven componentes de tipo material, formal, funcional e incluso social. De nuevo serán competentes para evaluar el relieve del valor patrimonial en este ámbito, los arquitectos especializados en la historia de la arquitectura militar, dentro de equipos multidisciplinares con asistencia de ingenieros militares, cartógrafos, geólogos y también sociólogos.

En la dimensión "documental", importarán factores propios de la geo-estrategia militar, que tendrán que ver con la inter-comunicación entre las diferentes torres de la red, o con el campo geográfico que cubría cada una en relación con su misión fundamental (avistar o repeler), pero también con la eficacia del diseño de la torre y de sus elementos defensivos en función de la defensa del lugar (guarniciones, baluartes, garitas, troneras...), incluso con las canteras que se abrieron para proveer los materiales utilizados en su ejecución. Por otra parte se atenderá también a la influencia con la geografía humana de la época en el entorno (localidades vecinas, población, actividades económicas...) y, a nivel general, con el contexto socio-político de entonces en el Antiguo Reino de Valencia. También pertenecerá a esta dimensión la toponimia, que en algunos casos se refiere a accidentes o reminiscencias del lugar (Sòl de Riu, Capicorb, Barranc, Pinaret, Grau Vell, Les Salines, Les Caletes, Xarco, Riu d'Aigües, l'Aigua Amarga...). Como es habitual en la dimensión documental de los valores patrimoniales, los grupos sociales receptores serán fundamentalmente los estudiosos del tema (especialistas e investigadores), aunque también podrá llegar a serlo cualquier individuo interesado si se le facilita la interpretación con medios divulgativos.

La dimensión "significativa" apuntará, sobre todo, a los valores paisajísticos de las torres, entendidos en este caso como el resultado de la interacción naturaleza / arquitectura en el territorio, tal y como la percibe la población. Visibilidad, presencia o cualidad del entorno serán factores determinantes de su valor, y no tanto en su condición geográfica (objetiva) sino sobre todo en su dimensión social y cultural (subjetiva). Desde el Convenio Europeo del Paisaje (Florencia, 2000) el atributo principal de su definición es la "percepción", y está asumido que su valor no reside en su condición objetiva como conjunción de objetos físicos, naturales y geográficos, sino en lo que los sujetos aprecian en ellos. Por eso, además de evaluar los primeros, será necesario rastrear los esquemas de valores y las expectativas, respecto a las torres en el paisaje, de quienes las disfrutan como bienes patrimoniales que serán, fundamentalmente, las gentes del lugar.

En cuanto a la dimensión "de uso" del valor patrimonial en el ámbito del entorno, habrá que buscarlo en su capacidad para convertirse en un recurso para el desarrollo cultural de la zona. Como elementos valorados, etas torres son susceptibles de ser convertidas en focos de atracción o lugares de encuentro de actividades. La clave está en encontrar el equilibrio entre la conservación de sus valores y la habilitación para los nuevos usos. Entre los condicionantes específicos del lugar estaría la masa crítica de actores, agentes y servicios, así como la existencia de una visión y estrategia compartida entre ellos: potencial de demanda, facilidad de comunicación, sinergias entre agentes locales, redes público-privadas, apoyo político y social... Los grupos sociales receptores de esta dimensión del valor patrimonial serán las gentes del lugar y potencialmente el turismo cultural.

- Aspecto 5. Tradiciones y técnicas

La convención UNESCO para la "Salvaguarda del Patrimonio Cultural Inmaterial" (2003) define el patrimonio inmaterial como "la parte no física de las tradiciones de un pueblo: expresiones culturales, idiomas, música, ceremonias, ritos, fiestas, maneras de hacer, actitudes de socialización, tradiciones orales..." El valor patrimonial de estos bienes concurre también en el reconocimiento de la identidad 
cultural por parte de los ciudadanos. Son cambiantes porque están en constante evolución y se protegen estudiándolos y documentándolos. El resto de bienes muebles e inmuebles vinculados con él le confieren valor y, al mismo tiempo, tienen valor mientras siguen teniendo utilidad para mantenerlo. Todos ellos conforman una "unidad cultural". Cualquier bien patrimonial es susceptible de lectura desde la unidad cultural a la que pertenece, aunque sea pretérita o extinta. Es el caso de la red de torres de defensa del litoral valenciano, que responde a tradiciones constructivas y militares del pasado, ya superadas y abandonadas. No obstante podrían llegar a ser re-vividas rememorándolas, por ejemplo, con estrategias museográficas. Estarán cualificados para la evaluación de este aspecto del valor patrimonial los técnicos en edificación especializados en historia de la construcción y los especialistas en historia militar, con la asistencia de antropólogos.

La dimensión "documental" de esta faceta del valor patrimonial residirá en los datos que permitan testimoniar tradiciones vinculadas con estas torres. Entre ellas estarían las tradiciones constructivas (materiales, técnicas y sistemas empleados en su construcción) y las técnicas militares asociadas tanto a la red como a las mismas torres y a cada uno de sus elementos de defensa. En ambos casos, junto a la interpretación de las prácticas a partir de los datos (algo así como "guías" sobre cómo proceder) deberán documentarse todos los elementos vinculados con ellas (armamentos, pertrechos, instrumentos, indumentarias...). También podría formar parte de esta dimensión la toponimia, que en algunos casos nos ilustra sobre trabajos, historias o relatos que dieron origen a su denominación (torre Nostra, del Rei, de la Corda, de la Mala Dona o la Renegada, de la Sal, l'Almadraba, del Guerro, del Descobridor, de la Galera, del Carabací, de l'Aguiló...). De nuevo los grupos sociales receptores serán fundamentalmente los estudiosos del tema (especialistas, investigadores...), aunque también podrá llegar a serlo cualquier individuo interesado, especialmente los vinculados al lugar.
En la dimensión "significativa", este valor inmaterial radicará en la en la posibilidad de recuperar los oficios o "saber hacer" de dichas tradiciones. En ser capaces de re-crearlas hoy, tal y como se hacían entonces. De este modo los "actores" se convertirían en portadores de una sabiduría con la que se identifican, que habrían heredado del pasado y podrían (y querrían) legar a las generaciones futuras. Los grupos sociales destinatarios de esta dimensión del valor patrimonial no serían tanto las gentes del lugar, sino los grupos vinculados con las tradiciones a recuperar: p.e. los sistemas constructivos tradicionales o las técnicas militares históricas.

La dimensión "de uso" se encontrará en su capacidad para albergar usos ligados a las tradiciones, celebraciones, festividades y demás ritos locales, sin contravenir el resto de valores patrimoniales. Fuegos artificiales, fiestas de moros y cristianos, pregones... son algunos de los ejemplos reales de re-utilización de estas torres relacionada con alguna tradición local. Es una práctica recurrente, que funciona como apropiación del bien por parte de la ciudadanía pero no siempre respeta su integridad o su cualidad patrimonial. Los grupos y comunidades locales serán sus principales receptores.

- Aspecto 6. Espíritu y sentimientos

El último de los aspectos enunciados en el documento de Nara es "espíritu y sentimientos". Tiene un carácter transversal. Participa de los demás aspectos, pero contemplados ahora desde una perspectiva social y emocional. No es susceptible de ser analizado en las tres dimensiones (documental, significativa y de uso) y por eso no forma parte de la matriz de valoración. Consiste en las relaciones afectivas entre bien y sociedad de pertenencia: apreciación, fruición, implicación, expectativas. $\mathrm{Su}$ intensidad se puede evaluar mediante estudios sociológicos de tipo subjetivo (encuestas y cuestionarios), aunque también se puede rastrear en las manifestaciones de la cultura local: literatura, pintura, canciones... Su evaluación correrá a cargo de sociólogos y requerirá estudios específicos, pormenorizados para cada ejemplar. 


\section{Conclusiones}

La Matriz de Valoración nos ha permitido apreciar y relacionar, de forma preliminar, los diferentes valores patrimoniales de la red de torres de defensa del litoral valenciano, en atención a la naturaleza diversa y transversal de dichos valores. Así mismo nos ha ayudado a señalar a los especialistas más capacitados para realizar la evaluación pormenorizada de cada uno de ellos, útil para conformar los equipos multidisciplinares encargados de calificarlos. Pero sobre todo nos ha llevado a identificar los grupos sociales destinados a disfrutarlos. Esta contribución será clave para planificar los procesos de participación, así como determinar los "horizontes de expectativa social", vinculados con cada valor, en relación con las diferentes torres y con el conjunto unitario que forma la red. En este trabajo apenas se ha alcanzado una visión general, resultado de un ejercicio eminentemente teórico o conceptual, cuya principal virtud ha sido la de establecer un procedimiento objetivo y estructurado para apreciar los valores culturales de estas torres, superando su complejidad intrínseca. Obviamente habrá de completarse con una serie de estudios temáticos, aspecto por aspecto, desarrollados con mayor profundidad por equipos transversales que atiendan a cada una de las tres dimensiones del valor patrimonial. La Matriz de Valoración se muestra como una herramienta de utilidad operativa para guiar y, sobre todo, priorizar la toma decisiones en la gestión y la intervención del patrimonio cultural.

\begin{tabular}{|c|c|c|c|}
\hline \multicolumn{4}{|c|}{ MATRIZ DE VALORACIÓN DE LAS TORRES DE DEFENSA DEL LITORAL VALENCIANO } \\
\hline & DOCUMENTAL & SIGNIFICATIVA & DE USO \\
\hline 1 MATERIA Y SUBSTANCIA & legibilidad & antigüedad & resiliencia \\
\hline 2 FORMA Y DISEÑO & tipología & estética & adaptabilidad \\
\hline 3 USO Y FUNCIÓN & hechos & iconicidad & interpretación \\
\hline 4 LOCALIZACIÓN Y CONTEXTO & geo-estrategia & paisajístico & desarrollo cultural \\
\hline 5 TRADICIONES Y TÉCNICAS & tradiciones & saber hacer & ritos locales \\
\hline ESPECIALISTAS & \multicolumn{3}{|c|}{ GRUPOS SOCIALES DESTINATARIOS DEL DIRFRUTE } \\
\hline 1 Técnicos en construcción & estudiosos & sociedad & técnicos \\
\hline 2 Arquitectos especializados & estudiosos & sociedad & usuarios \\
\hline 3 Arquitectos especializados & oriundos & grupos locales & sociedad \\
\hline 4 Arquitectos especializados & estudiosos & lugareños & turistas \\
\hline 5 Antropólogos & estudiosos & fieles & grupos locales \\
\hline
\end{tabular}

Fig. 3- Resultado de la Matriz de Valoración

\section{Notas}

El proyecto I+D "Torres de vigía y defensa del litoral valenciano. Generación de metadatos y modelos 3D para su interpretación y efectiva puesta en valor" cuenta con financiación del
Programa Estatal de Investigación Científica y Técnica de Excelencia, Subprograma Estatal de Generación de Conocimiento, promovido por el Ministerio de Economía y Competividad. Referencia HAR2013-41859-P.

\section{Referencias}

Consejo de Europa (2000). Convenio Europeo del Paisaje. Florencia. http://ipce.mcu.es/pdfs/convencion-florencia.pdf.

Eco, U. (2000). Los límites de la interpretación. Ed. Lumen. Barcelona.

Juan-Vidal, F. (2005). «Investigando en el Área de Expresión Gráfica Arquitectónica» in Investigando los Bienes Arquitectónicos. Ed. Ediciones Generales de la Construcción. Valencia.

Lemaire, $\mathrm{R}$ \& Stovel, $\mathrm{H}$ (1994). Documento de Nara en Autenticidad. Nara (Japón). http://ipce.mcu.es/pdfs/1994_Documento_Nara.pdf.

Lowenthal, D. (1985). The past is a foreing country. Cambridhe University Pres. Cambridge.

Muñoz Viñas, S. (2003). Teoría Contemporánea de la Restauración. Ed. Síntesis. Madrid.

UNESCO (2003). Convención para la Salvaguardia del Patrimonio Cultural Inmaterial. París. http://unesdoc.unesco.org/images/0018/001897/189761s.pdf. 